\title{
High level adaptive optics supervision software for fast transition to optimal performance
}

\section{Visa Korkiakoski, Francois Rigaut, Ian Price, Jesse Cranney, Francis Bennet, et al.}

Visa Korkiakoski, Francois Rigaut, lan Price, Jesse Cranney, Francis Bennet, Marcus Lingham, Nima Namjouyan, Doris Grosse, Michael Copeland, Celine d'Orgeville, "High level adaptive optics supervision software for fast transition to optimal performance," Proc. SPIE 10703, Adaptive Optics Systems VI, 107034T (30 July 2018); doi: 10.1117/12.2313080

Event: SPIE Astronomical Telescopes + Instrumentation, 2018, Austin, Texas, United States 


\title{
High level adaptive optics supervision software for fast transition to optimal performance
}

\author{
Visa Korkiakoski ${ }^{\mathrm{a}, \mathrm{b}}$, Francois Rigaut ${ }^{\mathrm{a}, \mathrm{b}}$, Ian Price ${ }^{\mathrm{a}, \mathrm{b}}$, Jesse Cranney ${ }^{\mathrm{c}, \mathrm{b}}$, Francis Bennet ${ }^{\mathrm{a}, \mathrm{b}}$, \\ Marcus Lingham ${ }^{\mathrm{a}, \mathrm{b}}$, Nima Namjouyan ${ }^{\mathrm{a}, \mathrm{b}}$, Doris Grosse ${ }^{\mathrm{a}, \mathrm{b}}$, Michael Copeland ${ }^{\mathrm{a}, \mathrm{b}}$, and Celine \\ d'Orgeville ${ }^{a, b}$ \\ ${ }^{a}$ Research School of Astronomy and Astrophysics, Australian National University, Mount \\ Stromlo Observatory, Weston Creek, Australia \\ ${ }^{\mathrm{b}}$ SERC Limited, Mount Stromlo, Weston Creek, Australia \\ ${ }^{c}$ University of Newcastle, Callaghan NSW 2308, Australia
}

\begin{abstract}
Space debris in low Earth orbit (LEO) below $1500 \mathrm{~km}$ is becoming an increasing threat to spacecrafts. To manage the threat, we are developing systems to improve the ground-based tracking and imaging of space debris and satellites. We also intend to demonstrate that it is possible to launch a high-power laser that modifies the orbits of the debris. However, atmospheric turbulence makes it necessary to use adaptive optics with such systems. When engaging with objects in LEO, the objects are available only a limited amount of time. During the observation window, the object has to be acquired and performance of all adaptive optics feedback loops optimised. We have implemented a high-level adaptive optics supervision tool to automatise time-consuming tasks related to calibration and performance monitoring. This paper describes in detail the current features of our software.
\end{abstract}

Keywords: generalised stereo-SCIDAR, atmospheric turbulence profile measurement, site characterisation, data post-processing

\section{INTRODUCTION}

Space debris in low Earth orbit (LEO) below $1500 \mathrm{~km}$ is becoming an increasing threat to spacecrafts. To manage the threat, we are developing systems to improve the tracking and imaging of space debris and satellites.

We also intend to demonstrate that it is possible to launch a high-power laser that modifies the orbits of the debris. ${ }^{1,2}$ However, atmospheric turbulence reduces the accuracy of tracking observations and spreads the uplink laser beam lessening its impact. Thus, adaptive optics is necessary to mitigate the issues caused by the turbulence.

Ground-based adaptive optics systems engaging with objects in LEO have a challenge: the objects are available only a limited amount of time. Per rotation, an observation time of only a few minutes is possible due to very fast angular speed of the objects.

During the observation window, the object has to be acquired, low-order AO loops closed, laser guide star pointed correctly, laser guide star acquired, all feedback control loops optimised and security measures for the high-power laser cleared; only then it is possible to engage the object.

Therefore, we have implemented a high-level adaptive optics supervision tool to automatise the time-consuming tasks related to calibration, performance optimisation and monitoring. The supervisor interfaces with the realtime computer that operates the actual hardware; it receives sequences of adaptive optics telemetry data, which are used to calculate optimal control parameters and visualise system performance for easy spotting of potential problems. This paper describes in detail the current features of our software and the experiments we have conducted to verify its proper performance.

Section 2 describes the general software platform that we are using. Then, section 3 elaborates the features that our AO supervisor currently has. Finally, section 4 presents the conclusions.

Further author information: (Send correspondence to Visa Korkiakoski)

Visa Korkiakoski: E-mail: visa.korkiakoski@anu.edu.au, Telephone: + 61261256084

Adaptive Optics Systems VI, edited by Laird M. Close, Laura Schreiber,

Dirk Schmidt, Proc. of SPIE Vol. 10703, 107034T · ( 2018 SPIE

CCC code: $0277-786 \mathrm{X} / 18 / \$ 18 \cdot$ doi: $10.1117 / 12.2313080$ 


\section{GENERAL SOFTWARE ARCHITECTURE}

This section gives a general overview of AO supervisor's software architecture.

We have tailored the supervisor for the two AO systems planned in near future: one for satellite imaging with either natural or laser guide stars (NGS, LGS), another one for applications where high-power laser is pre-compensated by the AO system and projected to space debris objects to modify their orbits. Currently, the supervisor supports NGS operations, but extending it to LGS mode is very straightforward.

As is typical for many other adaptive optics software platforms, we arrange the software in different layers, illustrated in Figure 1. The lowest layer is embedded within the hardware and typically comes along with the suppliers of deformable mirrors (DM) and detectors. The low-lever software layer contains the functionality required to communicate with the hardware as well as all the latency-critical tasks. The high-level software consists of the AO supervisor. It is in charge of most non-latency critical functionality: adjusting RTC parameters, visualising performance and streamlining operational tasks.

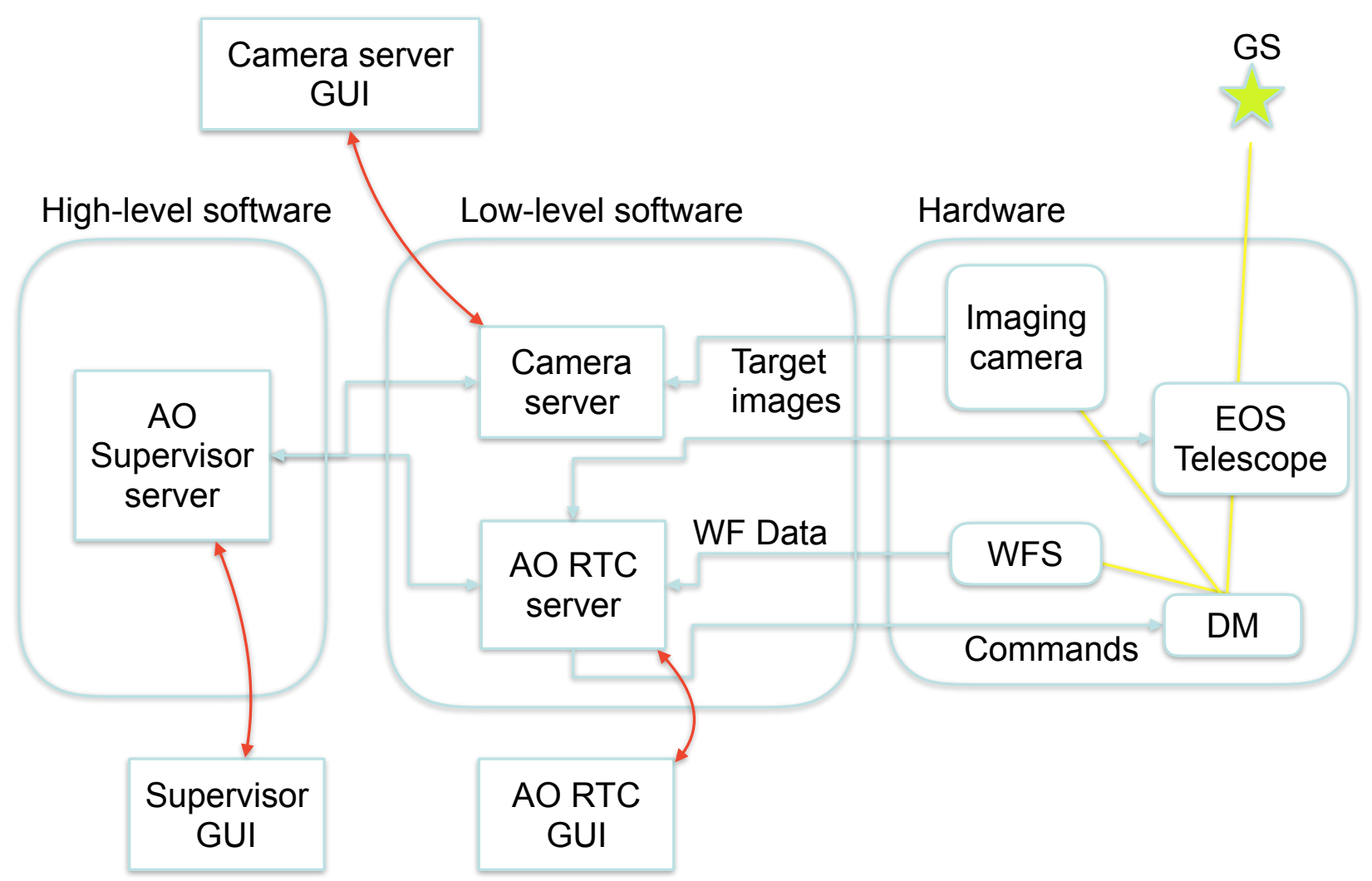

Figure 1: A schematic overview of our adaptive optics software platform.

Our low-level software contains camera server and AO RTC server. The former communicates with the imaging camera, adjusting its parameters, retrieving images and storing them for later use. The latter runs the adaptive optics system. It retrieves WFS detector data and uses it to calculate DM control voltages in real time. Our current implementation is capable of running at a rate of over $1 \mathrm{kHz}$. The camera server and AO RTC are 
implemented independently as different programs; they have no direct communication with each other and can be run on separate computers.

All servers, both low and high level, have their dedicated graphical user interfaces (GUI). The GUIs communicate with the servers in the same way as the AO supervisors communicates with the low-level software: we use TCP/IP network protocol with zeroqm. ${ }^{3}$

The low-level software is implemented using $\mathrm{C}++$ due to performance reasons.

The choice for the AO supervisor platform was driven by the need for a good support for intensive numerical calculations and graphical visualisations, as well as of ease of implementation and being comfortable for scientist user base. After briefly exploring options such as Processing IDE, Jython, we settled on implementing the supervisor using python and its extensive numerical libraries numpy and scipy, with user interfaces and graphical visualisations done by matplotlib, ${ }^{4} \mathrm{Qt}^{5}$ and pyqtgraph. ${ }^{6}$ So far, we have relied on the Anaconda distribution ${ }^{7}$ for all our high-level software components.

The AO supervisor uses threads to accomplish its tasks. It is possible to start the supervisor using python console, which remains responsive while supervisor carries out its activities. This way, a user has an access to the supervisor internal data structures, which can be inspected and modified at will by typing commands. While not the desired way to operate the system, the console makes is possible rapidly develop new features and debug the supervisor server.

The supervisor consist of the following threads:

- SequenceThread; requests AO telemetry sequences from RTC. (When requested, RTC saves a burst of telemetry on disk). Once SequenceThread sees that a telemetry sequence has been recorded by RTC, it copies the sequence for post-processing and notifies the other threads of the supervisor.

- SequenceProcessorThread; processes sequences of AO telemetry. Typically the computationally most intensive thread. Processing includes calculating PSDs of slope data and evaluating optimal control parameters for the RTC.

- ControlUpdateThread; communicates with RTC to adjust its control parameters, based on most recent information from the system.

- MonitorThread; monitors the general RTC performance. Keeps requesting snapshots of the RTC state. The retrieved snapshots will be used by other threads and GUI for various purposes.

- GUIWatchThread; monitors input from GUI, changes the supervisor parameters as requested by the user.

When testing the supervisor, we rely on different simulation modes. The RTC and camera servers have simulation modes that make it possible to to test software without having the hardware plugged in. In addition, we have added an additional RTC simulator module that is capable of simulating the RTC software with signals corresponding to realistic conditions, albeit with a frame rate of about 50 times slower compared to typical operations. The RTC simulator uses $\mathrm{yao}^{8}$ on backend to model atmospheric turbulence, light propagation to WFS detectors and DM shape after control voltages have been appleid.

Figure 2 contains a screenshot illustrating how the testing the supervisor with an RTC simulator typically looks like.

The screenshot contains five windows:

- AO supervisor console showing how the user has inspected slope variance in AO telemetry.

- RTC simulator console showing user inspecting simulated control signal.

- AO supervisor logging output.

- yao simulator window with incoming turbulence, DM status, WFS detector image, short exposure camera image and history of $\mathrm{AO}$ performance. 


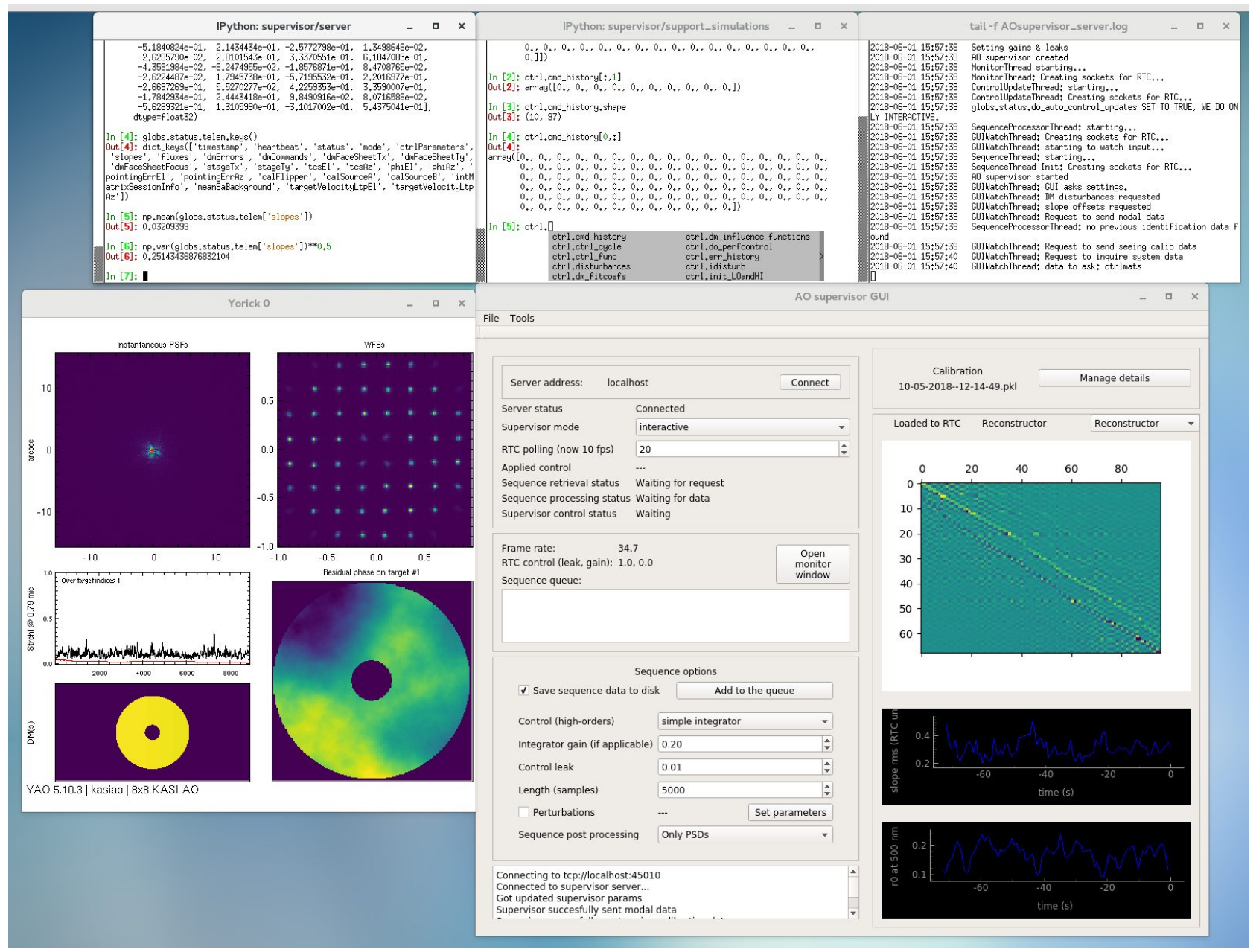

Figure 2: A screenshot illustrating testing the AO supervisor.

- AO supervisor GUI main window. Shows an overview of the supervisor status and gives the operator an easy access to most useful high-level AO operations.

The following section describes the AO supervisor features in more detail. 


\section{FEATURES}

\subsection{General operation principle}

The supervisor is designed to operate in batches. It collects a sequence of AO telemetry from RTC, processes it and adjusts control parameters accordingly. The operator can adjust parameters such as the length of the sequence, applied control strategy and control parameters such as simple integrator (SI) gain or leak. The next sequence is then recorded with updated control parameters.

The supervisor server also collects periodically, at a rate of about $10 \mathrm{~Hz}$, status messages from RTC. Those messages contain the essential status of the system: DM control voltages, measurement vectors, time stamps, control parameters, etc. The status information is processed and the supervisor GUI can present it in various way for operators to analyse it and spot potential problems.

\subsection{Supervisor calibration tools}

The supervisor includes tools to for calibration and system identification.

One of the most obvious function for the supervisor is calibration of DM - that is, finding the linear relation between measurement signal and the commands that need to be applied to cancel the corresponding wavefront. This consists of two relatively independent tasks: 1) calculating a command matrix that maps observed WFS signal to desired control voltages and 2) searching a reference signal that matches a flat wavefront towards imaging camera. The former is referred here simply as DM calibration while the latter is called non-common path (NCPA) calibration.

Figure 3 shows a screenshot illustrating the supervisor DM calibration tool. The tool lets the operator to select parameters a few inversion parameters as well as picking active subapertures - available flux is shown for guidance. The operator starts the calibration by pressing a button. This initiates a process where RTC pushes and pull a mirror modes similarly to standard AO calibration techniques for recording an interaction matrix. The used modes (as well as the time they are kept stable for measuring) is determined by the supervisor, although current implementation does not offer easy way for the operator to modify the values.

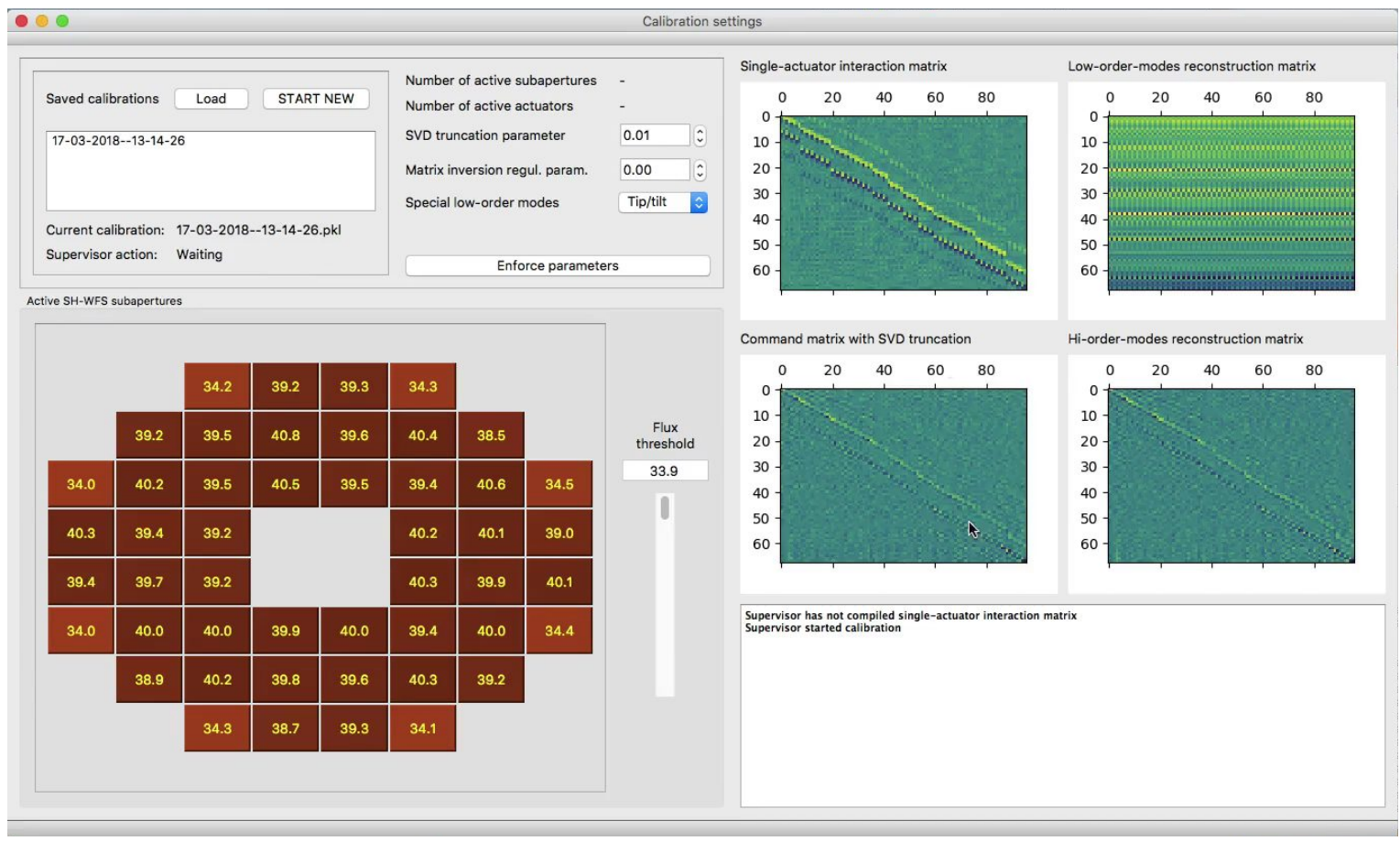

Figure 3: A screenshot showing AO supervisor DM calibration tool. 
After the interaction matrix recorded, supervisor calculates its pseudo-inverse to obtain the command matrix. The command matrix is then automatically uploaded to RTC and shown to the operator.

Figure 4 illustrates the supervisor's NCPA calibration tool. Currently, it implements a straightforward extremum seeking algorithm. Random DM disturbances are applied, maximum of the resulting imaging camera image is recorded, and an estimate of the NCPAs is updated. The algorithm is extremely robust, requires minimal assumptions about the system and works for a wide range of sources. However, it requires hundreds of iterations to converge.

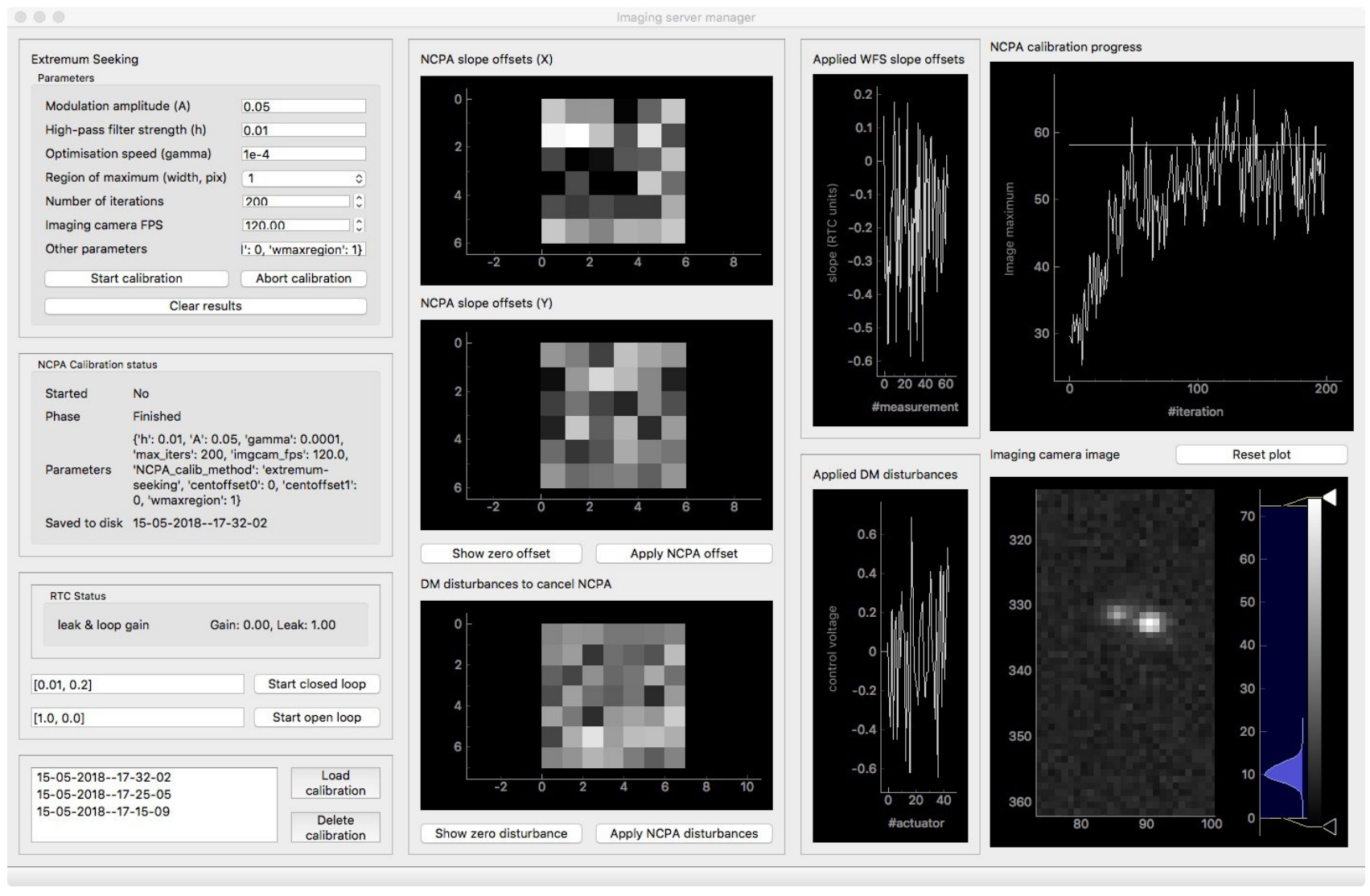

Figure 4: A screenshot showing AO supervisor NCPA calibration tool.

In addition, the supervisor has basic tools to aid in crude alignment and system testing. For instance, it lets user poke actuators individually via graphical users interface.

The supervisor also includes a tool to study the lag in the system. It asks RTC to introduce random DM disturbances, records the AO telemetry, calculates the noise transfer functions, finds the lag that best fit in the transfer functions, and visualises the whole process graphically for the operator.

\subsection{Real-time visualisation}

The supervisor has basic functionality to show users also some aspects of the real-time AO performance. This partly overlaps with what the RTC GUI does; the supervisor offers a more sophisticated view, however.

Figure 5 illustrates the real-time visualisation of the supervisor. That includes several tabs the operator can select, including a WFS view (image on the detector combined with SH-WFS slopes), slope maps arranged as a grid, DM commands arranged as a grid and projected to modes and DM errors (shown in the figure). 


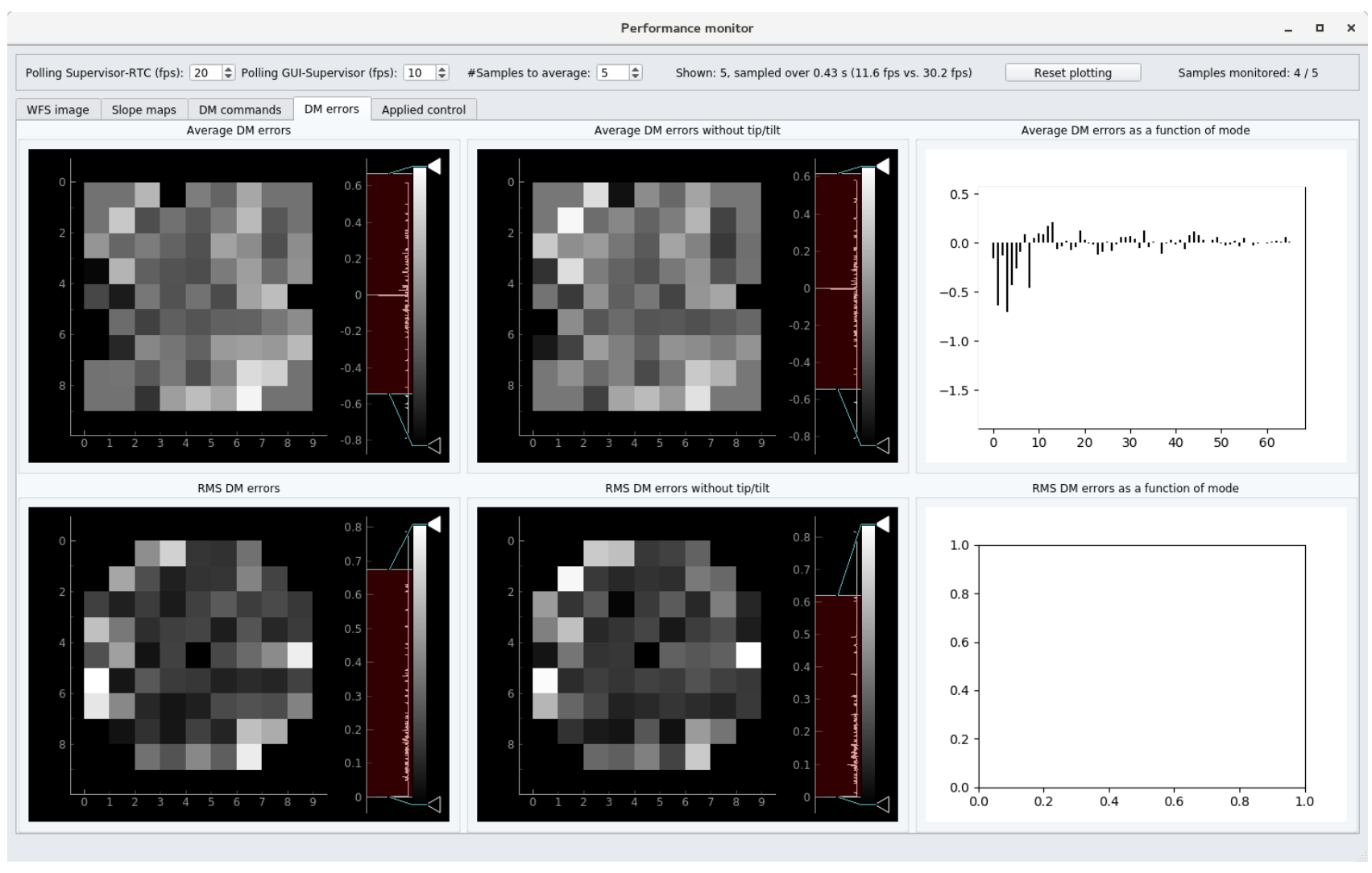

Figure 5: A screenshot illustrating the real-time visualisation capabilities of the supervisor.

The operator can choose to show the real-time monitor at any point, irrespectively to what the supervisor is doing. It is also possible to open several monitoring windows, if necessary to view the WFS slopes and DM commands at the same time.

\subsection{Control parameter tuning}

In its current manual mode, the AO operator needs to select which control strategy the supervisor should use. Current methods include simple integrator, optimal modal gain integrator (OMGI) ${ }^{9}$ and a single-layer predictive control (publication in progress).

If using a sophisticated control (other than simple integrator), the supervisor uses previous telemetry batch to estimate the optimal control parameters. Our current RTC implementation supports control laws that can be implemented as a matrix-vector multiplication followed by a dynamic controller (the simple integrator), followed by another matrix-vector multiplication applied on the actuator voltages.

Thus, for instance, when using OMGI, the supervisor calculates a new command matrix that incorporates the optimal loop gains and asks the RTC to use a gain value of one.

\subsection{Visualisation of processed AO telemetry}

The supervisor also includes an option to inspect the post-processed AO telemetry. This means that the following plots can be viewed by the supervisor GUI sequence comparison window:

- PSDs of closed-loop and pseudo-open loop WFS measurements. Plotting can be done in various spaces: slope space, modal space, eigenmode space. It is possible to select particular modes (such as tip, tilt or defocus), or average of several modes. Plotting scale can be chosen as log-log, lin-log or PSD*f vs f. 
- Transfer functions (as calculated by the comparison of open-loop and pseudo-open loop data)

- Phase structure functions

- Vibration identification. Tip and tilt vibrations are identified by searching peaks at the pseudo-open loop PSDs. An option is offered to modify parameters to tweak the vibration identification.

Figure 6 illustrates how the AO supervisor sequence comparison window looks like. The operator has selected two recorded telemetry sequences and plotted the PSDs of their tip-mode measurements.

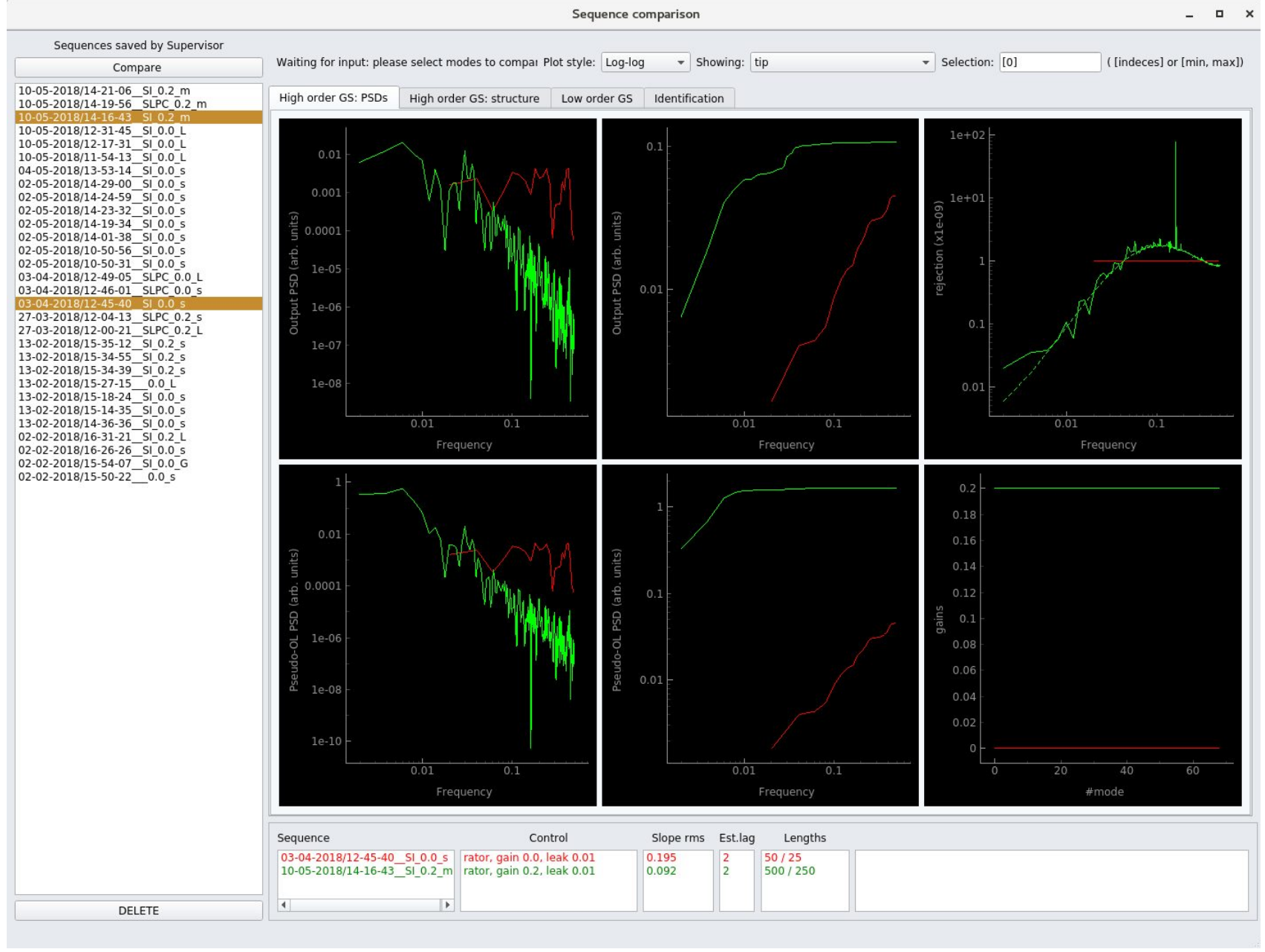

Figure 6: A screenshot illustrating the supervisor AO telemetry visualisation capabilities.

\section{CONCLUSIONS}

We have described the functionality of the high-level adaptive optics supervisor being developed for the applications of space debris engagement. The software streamlines basic operations thus making it possible for the $\mathrm{AO}$ operator to focus on the essentials, which is critical since the objects will be available only for a couple of minutes before passing behind the horizon.

The supervisor software employs modern tools and is written to friendly for AO scientists to debug and rapidly amend new features. 
Currently, the supervisor is tested mainly within a simulation environment. Our adaptive optics systems will be integrated in the coming months, thus requiring the commissioning of the AO supervisor by the end of 2018 .

\section{ACKNOWLEDGMENTS}

The authors would like to acknowledge the support of the Cooperative Research Centre for Space Environment Management (SERC Limited) through the Australian Government's Cooperative Research Centre Programme.

\section{REFERENCES}

[1] Bennet, F., Conan, R., D’Orgeville, C., Murray, M., Paulin, N., Price, I., Rigaut, F., Ritchie, I., Smith, C., and Uhlendorf, K., "Adaptive optics for laser space debris removal," in [SPIE Astronomical Telescopes+ Instrumentation], 844744-844744, International Society for Optics and Photonics (2012).

[2] Bennet, F., D’Orgeville, C., Price, I., Rigaut, F., Ritchie, I., and Smith, C., "Adaptive optics for satellite imaging and space debris ranging," in [Proceedings of the Advanced Maui Optical and Space Surveillance Technologies Conference], Ryan, S., ed., 1, 2, The Maui Economic Development Board (2015).

[3] Hintjens, P., [ZeroMQ: messaging for many applications], " O'Reilly Media, Inc." (2013).

[4] Hunter, J. D., "Matplotlib: A 2d graphics environment," Computing in science \& engineering 9(3), 90-95 (2007).

[5] Summerfield, M., [Advanced Qt Programming: Creating Great Software with C++ and Qt 4], Pearson Education (2010).

[6] Campagnola, L., "Pyqtgraph-scientific graphics and gui library for python," (2016).

[7] Analytics, C., "Anaconda: Completely free enterprise-ready python distribution for large-scale data processing, predictive analytics, and scientific computing."

[8] Rigaut1a, F. and Van Dam, M., "Simulating astronomical adaptive optics systems using yao," (2013).

[9] Gendron, E. and Léna, P., "Astronomical adaptive optics. 1: Modal control optimization," Astronomy and Astrophysics 291, 337-347 (1994). 